\title{
Mineralocorticoid receptor antagonists for heart failure: systematic review and meta-analysis
}

\author{
Nicolas M. Berbenetz and Marko Mrkobrada*
}

\begin{abstract}
Background: Mineralocorticoid receptor antagonists (MRAs) have been associated with improved patient outcomes in patients with heart failure with reduced ejection fraction (HFrEF) but not preserved ejection fraction (HFpEF). We conducted a systematic review and meta-analysis of selective and nonselective MRAs in HFrEF and HFpEF.

Methods: We searched Cochrane Central Register of Controlled Trials, MEDLINE and EMBASE. We included randomized controlled trials (RCT) of MRAs in adults with HFpEF or HFrEF if they reported data on major adverse cardiac events or drug safety.

Results: We identified 15 studies representing 16321 patients. MRAs were associated with a reduced risk of cardiovascular death (RR 0.81 [0.75-0.87], I I $^{2} \%$ ), all-cause mortality (RR 0.83 [0.77-0.88], I I $^{2} \%$ ), and cardiac hospitalizations (RR 0.80 [0.70-0.92], $I^{2}$ 58.4\%). However, an a-priori specified subgroup analysis demonstrated that these benefits were limited to HFrEF (cardiovascular death RR 0.79 [0.73-0.86], $\mathrm{I}^{2}$ 0\%; all-cause mortality RR 0.81 [0.75-0.87], I $I^{2}$ \%; cardiac hospitalizations RR 0.76 [0.64-0.90], I ${ }^{2}$ 68\%), but not HFpEF (all-cause mortality RR 0.92 [0.79-1.08], I $I^{2}$ 0\%; cardiac hospitalizations RR 0.91 [0.67-1.24], I ${ }^{2}$ 17\%). MRAs increased the risk of hyperkalemia (RR 2.03 [1.78-2.31], I $I^{2}$ \%). Nonselective MRAs, but not selective MRAs increased the risk of gynecomastia (RR 7.37 [4.42-12.30], I $I^{2} 0 \%$ vs. RR 0.74 [0.43-1.27], I $I^{2} 0 \%$ ). Evidence was of moderate quality for cardiovascular death, all-cause mortality and cardiovascular hospitalizations; and high-quality for hyperkalemia and gynecomastia.
\end{abstract}

Conclusions: MRAs reduce the risk of adverse cardiac events in HFrEF but not HFpEF. MRA use in HFpEF increases the risk of harm from hyperkalemia and gynecomastia. Selective MRAs are equally effective as nonselective MRAs, without a risk of gynecomastia.

Keywords: Heart failure, Heart failure with reduced ejection fraction, Heart failure with preserved ejection fraction, Mineralocorticoid receptor antagonists, Systematic review

\section{Background}

Heart failure (HF) has significant morbidity and is often a result of impaired left ventricular myocardial function [1]. HF with preserved ejection fraction (HFpEF) involves impaired myocardial function with normal left ventricle size and ejection fraction; in contrast, HF with reduced ejection fraction (HFrEF) involves an enlarged left ventricle size and reduced ejection fraction. Evidence-based HF treatment reduces morbidity and

\footnotetext{
* Correspondence: mmrkobr@uwo.ca

Department of Medicine, Western University, 339 Windermere Road, London, ON N6A 5A5, Canada
}

mortality in HFrEF [2]. HFpEF prevalence is rising due to an ageing population, however, there are no treatments which reduce morbidity and mortality [3]. Diagnosing HFpEF is often confounded by the occurrence of similar symptoms in patients with multiple medical comorbidities [3]. The most prevalent risk factor for HFpEF is hypertension [3]. Several RCTs have explored the benefits of $\beta$-blockers [4], ARBs [5], ACEi [6], and mineralocorticoid receptor antagonists (MRAs) [7] in $\mathrm{HFpEF}$ and identified trends towards reduced cardiovascular morbidity and mortality [8]. The lack of strong 
evidence in HFpEF treatment has led to considerable treatment variation [9].

MRAs can be selective (e.g., eplerenone) or nonselective (e.g., spironolactone). Eplerenone was synthesized through chemical modification of spironolactone in order to enhance binding of mineralocorticoid receptors while reducing off-target binding to progesterone or androgen receptors [10]. Eplerenone is associated with lower rates of impotence, gynecomastia or breast pain in comparison to spironolactone $[11,12]$.

MRAs found initial use in HF exacerbations as diuretics in patients' refractory to combined ACEi and loop diuretic therapy [13]. However, spironolactone at doses with no significant diuretic effect was found to reduce cardiovascular mortality [14]. This effect was presumably due to a reduction in myocardial and vascular fibrosis [14]. This effect may arise from spironolactone blocking aldosterone's ability to stimulate collagen synthesis at the myocardial level [15]. Spironolactone and eplerenone have demonstrated significant mortality benefit in $\operatorname{HFrEF}[11,12]$. In contrast, MRAs in HFpEF do not reduce all-cause mortality, however, they do reduce hospitalizations, improve quality of life, and improve echocardiographic measurements of diastolic function [16].

Chronically elevated aldosterone levels contribute towards structural changes in the heart which promote water retention, myocardial fibrosis, and increased arrhythmogenicity [17]. MRAs in HFpEF improved echocardiographic and biochemical measures of diastolic function [16, 18]. However, a large prospective RCT in HFpEF patients treated with spironolactone did not demonstrate a significant benefit in terms of cardiovascular outcomes [7].

\section{Objectives}

Our objectives were to evaluate the risks and benefits of MRA usage in adults with HF. We were particularly interested in differences between selective and nonselective MRAs in HFpEF and HFrEF in terms of cardiovascular outcomes and adverse effects.

\section{Methods}

Our systematic review and meta-analysis complies with the PRISMA statement [19].

\section{Eligibility criteria}

We included randomized controlled trials (RCTs) of MRAs vs. placebo or standard therapy in adults ( $\geq 18$ years old) with HFpEF or HFrEF. Included trials evaluated nonselective MRAs (e.g., canrenone, spironolactone), and selective MRAs (e.g., eplerenone, finerenone). Included trials contained at least one outcome of interest: mortality (all-cause or cardiovascular), cardiovascular hospitalizations, hyperkalemia, or gynecomastia.

\section{Literature search}

We searched the Cochrane Central Register of Controlled Trials (The Cochrane Library Issue 1, 2016), MEDLINE (January 1995 to January 29, 2016), and EMBASE (January 1995 to January 29, 2016) for articles meeting our inclusion criteria. Our search strategy for Ovid MEDLINE and EMBASE is in Appendix 1 and our search strategy for the Cochrane Register of Controlled Trials is in Appendix 2. Our search did not have any language restrictions. We excluded reviews, editorials, and conferences but not unpublished studies or abstracts.

\section{Study selection}

We entered the retrieved citations into Reference Manager (v12.0.3), and duplicate records were removed. One investigator (NB) screened citations for relevance based on their title and abstract. Both investigators reviewed the full text articles of relevant articles for study inclusion. Cohen's kappa statistic was used to quantify chance-corrected agreement between the investigators. Disagreements on study inclusion were resolved through a consensus process of having a discussion between the two investigators.

\section{Data collection and analysis}

Both investigators extracted data independently from included articles. We resolved disagreements during data extraction by consensus. If data were incomplete or unclear we attempted to contact trial authors. We extracted the following items from each study: population (type of heart failure, study size), intervention (MRA type), control (placebo, none, other), and outcomes (all-cause mortality, cardiovascular mortality, hospitalizations, hyperkalemia, and gynecomastia/breast pain). We used each study's definition of these outcomes.

\section{Risk of bias}

Our risk of bias assessment was completed using the Cochrane Risk of Bias Tool. It evaluates individual studies for several biases: selection, performance, detection, attrition and reporting. We evaluated the quality of evidence for each outcome using GRADE criteria [20], which evaluates an outcome across studies based on risk of bias, inconsistency, indirectness, imprecision and publication bias.

\section{Statistical analysis}

We obtained the relative risk for each outcome from the original study and used RevMan (version 5.3.5) and $\mathrm{R}$ 
$[21,22]$ to analyze data and generate figures. We used the Mantel-Haenszel method with a 95\% confidence interval, and a random effects model to pool results. We quantified statistical heterogeneity using the $\mathrm{I}^{2}$ statistic. We interpreted an $\mathrm{I}^{2}$ value of $0-25 \%$ as low heterogeneity, $25-50 \%$ as moderate heterogeneity, and $>50 \%$ as high heterogeneity. A priori we established two hypotheses to explain potential heterogeneity: HF type (HFpEF and HFrEF), and MRA type (selective, or nonselective). We assessed for publication bias using funnel plots for each outcome.

\section{Results}

\section{Trial selection}

We screened 2566 citations, and selected 36 for full text review. Of these, 15 articles [7, 11, 12, 18, 23-33] met our inclusion criteria and were included in our systematic review (see Fig. 1). Overall, there was excellent agreement on trial eligibility (Cohen's kappa 94\%). We excluded articles from the systematic review because of treatment in a non-HF setting $(N=4)$, lack of relevant outcomes $(N=13)$, study duplication $(N=3)$, and not an RCT design $(N=1)$.

\section{Trial characteristics}

Table 1 reports the trial characteristics of the 15 RCTs containing 16321 patients. The patients had either HFpEF $(N=4027)$ or HFrEF $(N=12294)$ and the MRA treatment group was either nonselective, e.g., canrenone, spironolactone, $N=11$ RCTs, 6678 patients; or selective, e.g., eplerenone, $N=4$ RCTs, 9643 patients. Studies had an average length of follow-up of 15 months.

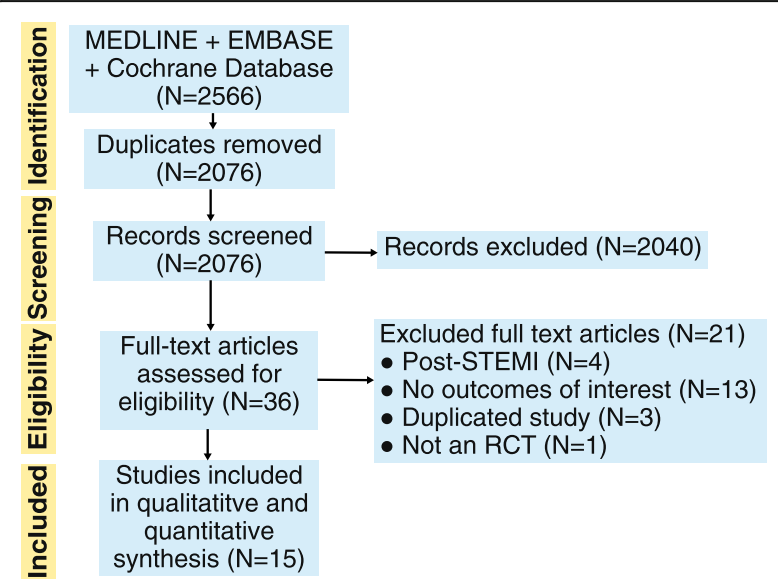

Fig. 1 Study selection flow diagram. Overview of process used to identify studies for inclusion in the systematic review. Three databases (MEDLINE, EMBASE, Cochrane) were searched for relevant articles. After identification, studies were screened against our inclusion criteria. Included studies were used in our meta-analysis

\section{Risk of bias within included trials}

Table 2 reports the quality of included studies. Five trials had unclear or absent allocation concealment $[23,25,26,28,30]$. Two studies had inadequate blinding and were of single-blind design [23, 32]. Two large studies were terminated early due to meeting pre-defined benefit criteria [11,33]. Another two studies did not use intention-to-treat analysis. Overall, loss-to-follow-up was low with a range of 0 to $6.6 \%$.

\section{Results of meta-analysis}

Table 3 reports a summary of findings. We included outcomes for cardiovascular death (7 RCTs), all-cause mortality (12 RCTs), cardiac hospitalization (10 RCTs), hyperkalemia (15 RCTs), and gynecomastia $(N=11$ RCTs). Quality of evidence for cardiovascular death, allcause mortality, and cardiac hospitalization were rated moderate; hyperkalemia and gynecomastia were rated high using GRADE guidelines [20]. For each outcome, HFrEF evidence was of high quality, but the quality of evidence for HFpEF was of moderate quality for allcause mortality, cardiovascular death, and cardiac hospitalizations.

Meta-analysis of cardiovascular death (see Fig. 2) revealed a significant risk reduction, RR 0.81 [0.75-0.87], $\mathrm{I}^{2} 0 \%$ (low heterogeneity). Our analysis of cardiovascular death by HF type indicated only a single trial of HFpEF (TOPCAT) which had no significant reduction in cardiovascular death [7]. Using either selective or nonselective MRA had a similar reduction in cardiovascular death (Additional file 1: Figure S1).

Meta-analysis of all-cause mortality (see Fig. 3) revealed a significant risk reduction, RR 0.83 [0.770.88 ], $\mathrm{I}^{2} 0 \%$ (low heterogeneity). HF type subgroups indicated the benefit was limited to HFrEF. Use of either a selective or nonselective MRA had a similar reduction in all-cause mortality (Additional file 2: Figure S2).

Meta-analysis of cardiac hospitalizations (see Fig. 4) revealed a significant risk reduction, RR 0.80 [0.700.92], $\mathrm{I}^{2} 58.4 \%$ (high heterogeneity). Our a priori subgroup analysis partially explained the heterogeneity within this outcome, as a significant reduction in cardiac hospitalizations was found in the HFrEF and nonselective MRA subgroups (Additional file 3: Figure S3).

Hyperkalemia was significantly more common with MRA use, RR 2.03 [1.78-2.31], I ${ }^{2}$ 0\% (low heterogeneity), see Fig. 5. Subgroup analysis by MRA or HF type did not significantly influence the rate of hyperkalemia (Additional file 4: Figure S4).

Gynecomastia was significantly more common with MRA use, RR 3.28 [1.18-9.10], $\mathrm{I}^{2}$ 81.7\% (high heterogeneity), see Fig. 6. MRA type explained this heterogeneity 
Table 1 Overview of trials meeting systematic review inclusion criteria

\begin{tabular}{|c|c|c|c|c|c|c|c|}
\hline Author & Year & Population & $\operatorname{Exp}(N)$ & Cont (N) & Intervention & Drug dose & Follow-up (months) \\
\hline Akbulut & 2003 & HFrEF, EF $\leq 35 \%, N Y H A ~ I I I$ & 35 & 35 & spironolactone & 25 mg daily & 3 \\
\hline Boccanelli & 2009 & HFrEF, EF $\leq 45 \%, \mathrm{NYHA} \|$ & 215 & 223 & canrenone & 25 mg daily & 12 \\
\hline Chan & 2007 & HFrEF, EF < 40\%, NYHA I-III & 23 & 25 & spironolactone & 25 mg daily & 12 \\
\hline Cicoira & 2002 & HFrEF, EF $\leq 45 \%$, NYHA III & 54 & 52 & spironolactone & 25 mg daily & 12 \\
\hline Deswal & 2011 & HFpEF, EF $\geq 50 \%$, NYHA II-III & 25 & 23 & eplerenone & 25 mg daily & 6 \\
\hline Edelmann & 2013 & HFpEF, EF $\geq 50 \%$, NYHA II-III & 213 & 209 & spironolactone & 25 mg daily & 12 \\
\hline Edwards & 2009 & HFpEF, CKD stage 2-3 & 56 & 56 & spironolactone & 25 mg daily & 9 \\
\hline Zannad & 2011 & HFrEF, EF $\leq 35 \%$, NYHA $\|$ & 1364 & 1373 & eplerenone & 25-50 mg daily & 21 \\
\hline Pitt & 2003 & $\mathrm{Ml}+\mathrm{HFrEF}, \mathrm{EF} \leq 40 \%$ & 3319 & 3313 & eplerenone & 25-50 mg daily & 16 \\
\hline Gao & 2007 & HFrEF, EF < 45\%, NYHA II-IV & 58 & 58 & spironolactone & 20 mg daily & 6 \\
\hline Pitt & 2013 & HFrEF, EF $\leq 40 \%$, CKD stage $2-3$ & 63 & 65 & spironolactone & 25-50 mg daily & 1 \\
\hline Pitt & 1999 & HFrEF, EF < 35\%, NYHA III-IV & 822 & 841 & spironolactone & 25-50 mg daily & 24 \\
\hline Pitt & 2014 & $\mathrm{HFpEF}, \mathrm{EF} \geq 45 \%$ & 1722 & 1723 & spironolactone & 15-45 mg daily & 40 \\
\hline Udelson & 2010 & HFrEF, EF $\leq 35 \%$ NYHA II-III & 117 & 109 & eplerenone & 50 mg daily & 9 \\
\hline Vizzardi & 2014 & $\mathrm{HFrEF}, \mathrm{EF}<40 \%$, NYHA I-II & 65 & 65 & spironolactone & 25-100 mg daily & 44 \\
\hline
\end{tabular}

as selective MRAs did not produce significant amounts of gynecomastia (RR 0.74 [0.43-1.27], $\mathrm{I}^{2} 0 \%$ ) while nonselective MRAs did (RR 7.37 [4.42-12.30], $\left.\mathrm{I}^{2} 0 \%\right)$.

Our analysis of funnel plots for each outcome except gynecomastia revealed no significant asymmetry (Additional file 5: Figure S5, Additional file 6: Figure S6, Additional file 7: Figure S7, Additional file 8: Figure S8 and Additional file 9: Figure S9) and suggested the absence of publication bias. Two MRA subgroups within the funnel plot for gynecomastia explained the asymmetry of the plot (Additional file 9: Figure S9).

\section{Discussion}

\section{Summary of evidence}

15 trials evaluated the use of MRAs compared to placebo or no treatment for HF. MRA use in patients with heart failure was associated with a significant reduction in adverse cardiovascular outcomes: cardiovascular death (RR 0.81 [0.75-0.87], $\mathrm{I}^{2} 0 \%$ ), all-cause

Table 2 Risk of bias summary for each study included in the meta-analysis

\begin{tabular}{|c|c|c|c|c|c|c|}
\hline Author & Year & Allocation concealment & Blinding & Intention to treat analysis & Loss to follow-up (\%) & Early trial termination \\
\hline Akbulut & 2003 & Unclear & No & Yes & 0.0 & No \\
\hline Boccanelli & 2009 & Yes & Yes & Yes & 6.2 & No \\
\hline Chan & 2007 & Unclear & Yes & Yes & 0.0 & No \\
\hline Cicoira & 2002 & Unclear & Yes & Yes & 6.6 & No \\
\hline Deswal & 2011 & Yes & Yes & No & 4.3 & No \\
\hline Edelmann & 2013 & Yes & Yes & Yes & 1.2 & No \\
\hline Edwards & 2009 & Unclear & Yes & No & 2.7 & No \\
\hline Zannad & 2011 & Yes & Yes & Yes & 1.2 & Yes \\
\hline Pitt & 2003 & Yes & Yes & Yes & 0.3 & No \\
\hline Gao & 2007 & Yes & Yes & Yes & 0.0 & No \\
\hline Pitt & 2013 & No (open label Aldactone) & Yes & Yes & 0.0 & No \\
\hline Pitt & 1999 & Yes & Yes & Yes & 0.0 & Yes \\
\hline Pitt & 2014 & Yes & Yes & Yes & 3.8 & No \\
\hline Udelson & 2010 & Yes & Yes & Yes & 0.0 & No \\
\hline Vizzardi & 2014 & Yes & No & Yes & 0.0 & No \\
\hline
\end{tabular}


Table 3 Summary of findings for the effect of mineralocorticoid receptor antagonists in treating Heart Failure

\begin{tabular}{|c|c|c|c|c|c|}
\hline \multirow[t]{2}{*}{ Outcome } & \multirow{2}{*}{$\begin{array}{l}\text { № of } \\
\text { participants } \\
\text { (studies) }\end{array}$} & \multirow{2}{*}{$\begin{array}{l}\text { Quality of } \\
\text { the } \\
\text { evidence } \\
\text { (GRADE) }\end{array}$} & \multirow{2}{*}{$\begin{array}{l}\text { Relative effect } \\
(95 \% \mathrm{Cl})\end{array}$} & \multicolumn{2}{|c|}{ Anticipated absolute effects } \\
\hline & & & & Risk with placebo & Risk difference with MRA \\
\hline Cardiovascular death & $\begin{array}{l}15115 \\
\text { (7 RCTs) }\end{array}$ & $\begin{array}{l}\oplus \oplus \oplus \\
\text { MODERATE }\end{array}$ & $\begin{array}{l}\text { RR } 0.81 \\
(0.75 \text { to } 0.87)\end{array}$ & 155 per 1000 & $\begin{array}{l}29 \text { fewer per } 1000 \\
\text { (39 fewer to } 20 \text { fewer) }\end{array}$ \\
\hline Cardiovascular death - rEF & $\begin{array}{l}11670 \\
\text { (6 RCTs) }\end{array}$ & $\begin{array}{l}\oplus \oplus \oplus \oplus \\
\mathrm{HIGH}\end{array}$ & $\begin{array}{l}\text { RR } 0.79 \\
(0.73 \text { to } 0.86)\end{array}$ & 171 per 1000 & $\begin{array}{l}36 \text { fewer per } 1000 \\
\text { (46 fewer to } 24 \text { fewer) }\end{array}$ \\
\hline Cardiovascular death - pEF & $\begin{array}{l}3445 \\
(1 \mathrm{RCT})\end{array}$ & $\begin{array}{l}\oplus \oplus \oplus \\
\text { MODERATE }\end{array}$ & $\begin{array}{l}\text { RR } 0.91 \\
\text { (0.74 to 1.11) }\end{array}$ & 102 per 1000 & $\begin{array}{l}9 \text { fewer per } 1000 \\
\text { (27 fewer to } 11 \text { more) }\end{array}$ \\
\hline All cause mortality & $\begin{array}{l}15919 \\
\text { (12 RCTs) }\end{array}$ & $\begin{array}{l}\oplus \oplus \oplus \\
\text { MODERATE }^{c}\end{array}$ & $\begin{array}{l}\text { RR } 0.83 \\
\text { (0.77 to } 0.88)\end{array}$ & 182 per 1000 & $\begin{array}{l}31 \text { fewer per } 1000 \\
\text { ( } 42 \text { fewer to } 22 \text { fewer) }\end{array}$ \\
\hline All cause mortality - rEF & $\begin{array}{l}11892 \\
\text { (8 RCTs) }\end{array}$ & $\begin{array}{l}\oplus \oplus \oplus \oplus \\
H I G H\end{array}$ & $\begin{array}{l}\text { RR } 0.81 \\
\text { ( } 0.75 \text { to } 0.87)\end{array}$ & 197 per 1000 & $\begin{array}{l}38 \text { fewer per } 1000 \\
\text { (49 fewer to } 26 \text { fewer) }\end{array}$ \\
\hline All cause mortality - pEF & $\begin{array}{l}4027 \\
\text { (4 RCTs) }\end{array}$ & $\begin{array}{l}\oplus \oplus \oplus \\
\text { MODERATE }\end{array}$ & $\begin{array}{l}\text { RR } 0.92 \\
\text { (0.79 to } 1.08)\end{array}$ & 136 per 1000 & $\begin{array}{l}11 \text { fewer per } 1000 \\
\text { (29 fewer to } 11 \text { more) }\end{array}$ \\
\hline Cardiac hospitalization & $\begin{array}{l}15669 \\
\text { (10 RCTs) }\end{array}$ & $\begin{array}{l}\oplus \oplus \oplus \\
\text { MODERATE }\end{array}$ & $\begin{array}{l}\text { RR } 0.80 \\
\text { (0.70 to } 0.92)\end{array}$ & 217 per 1000 & $\begin{array}{l}43 \text { fewer per } 1000 \\
\text { (65 fewer to } 17 \text { fewer) }\end{array}$ \\
\hline Cardiac hospitalization - rEF & $\begin{array}{l}11754 \\
\text { (7 RCTs) }\end{array}$ & $\begin{array}{l}\oplus \oplus \oplus \\
\text { MODERATE }\end{array}$ & $\begin{array}{l}\text { RR } 0.76 \\
\text { (0.64 to } 0.90)\end{array}$ & 245 per 1000 & $\begin{array}{l}59 \text { fewer per } 1000 \\
\text { (88 fewer to } 24 \text { fewer) }\end{array}$ \\
\hline Cardiac hospitalization - pEF & $\begin{array}{l}3915 \\
\text { (3 RCTs) }\end{array}$ & $\begin{array}{l}\oplus \oplus \oplus \\
\text { MODERATE }\end{array}$ & $\begin{array}{l}\text { RR } 0.91 \\
\text { (0.67 to } 1.24)\end{array}$ & 134 per 1000 & $\begin{array}{l}12 \text { fewer per } 1000 \\
\text { (44 fewer to } 32 \text { more) }\end{array}$ \\
\hline Hyperkalemia & $\begin{array}{l}16321 \\
(15 \mathrm{RCTs})\end{array}$ & $\begin{array}{l}\oplus \oplus \oplus \oplus \\
H I G H\end{array}$ & $\begin{array}{l}\text { RR } 2.03 \\
\text { (1.78 to } 2.31 \text { ) }\end{array}$ & 37 per 1000 & $\begin{array}{l}39 \text { more per } 1000 \\
\text { ( } 29 \text { more to } 49 \text { more) }\end{array}$ \\
\hline Gynecomastia or breast pain - nonselective & $\begin{array}{l}6432 \\
\text { (8 RCTs) }\end{array}$ & $\begin{array}{l}\oplus \oplus \oplus \oplus \\
H I G H\end{array}$ & $\begin{array}{l}\text { RR } 7.37 \\
\text { (4.42 to } 12.30 \text { ) }\end{array}$ & 5 per 1000 & $\begin{array}{l}30 \text { more per } 1000 \\
\text { (16 more to } 53 \text { more) }\end{array}$ \\
\hline Gynecomastia or breast pain - selective & $\begin{array}{l}9417 \\
\text { (3 RCTs) }\end{array}$ & $\begin{array}{l}\oplus \oplus \oplus \oplus \\
\mathrm{HIGH}\end{array}$ & $\begin{array}{l}\text { RR } 0.74 \\
\text { (0.43 to } 1.27)\end{array}$ & 7 per 1000 & $\begin{array}{l}2 \text { fewer per } 1000 \\
\text { ( } 4 \text { fewer to } 2 \text { more) }\end{array}$ \\
\hline
\end{tabular}

Cl Confidence interval, $R R$ Risk ratio

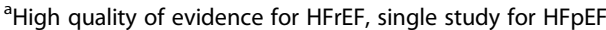

${ }^{b}$ Single trial with confidence interval which crossed unity

'High quality of evidence for HFrEF, moderate quality evidence for HFpEF

${ }^{\mathrm{d} C o n f i d e n c e ~ i n t e r v a l ~ o f ~ d a t a ~ c r o s s e d ~ u n i t y ~}$

mortality (RR 0.83 [0.77-0.88], $\mathrm{I}^{2} 0 \%$ ), and cardiac hospitalizations (RR 0.80 [0.70-0.92], I $\mathrm{I}^{2} 58.4 \%$ ). Our a priori specified subgroup analysis demonstrated that the benefits of MRAs are limited to HFrEF. Both selective and nonselective MRAs increase the risk of hyperkalemia (RR $\left.2.03 \quad[1.78-2.31], \mathrm{I}^{2} \quad 0 \%\right)$, but gynecomastia is limited to nonselective MRAs (nonselective MRAs RR 7.37 [4.42-12.30], $\mathrm{I}^{2} 0 \%$ vs. selective $\begin{array}{lllllllll}\text { MRAs } & \mathrm{RR} & 0.74 & {[0.43-1.27],} & \mathrm{I}^{2} & 0 \% \mathrm{RR} & 7.37 & \text { [4.42- }\end{array}$ 12.30).

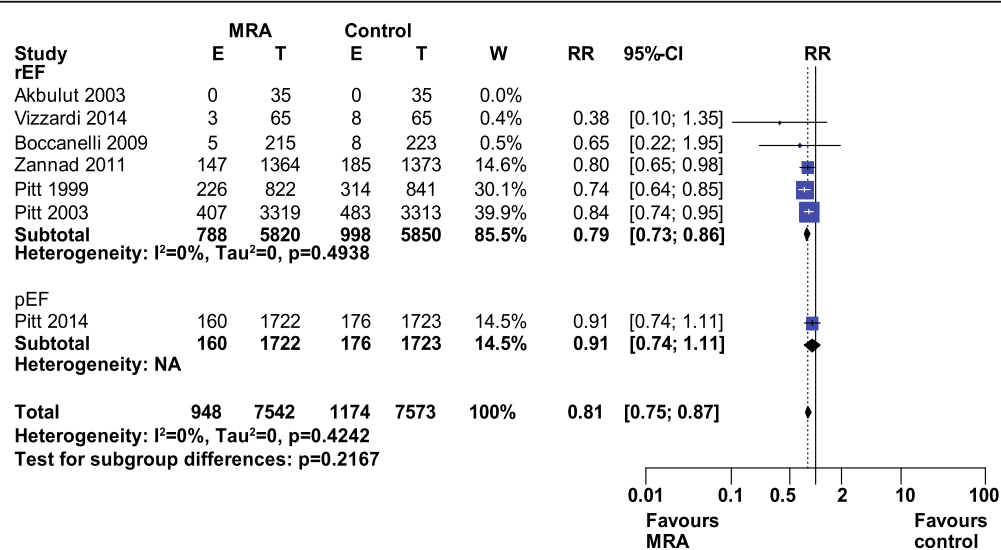

Fig. 2 Forest plot of cardiovascular death with MRA use in HF. Seven trials reported cardiovascular death rates when using MRAs in HF compared to control. Our Forest plot has been subdivided according to HF type 


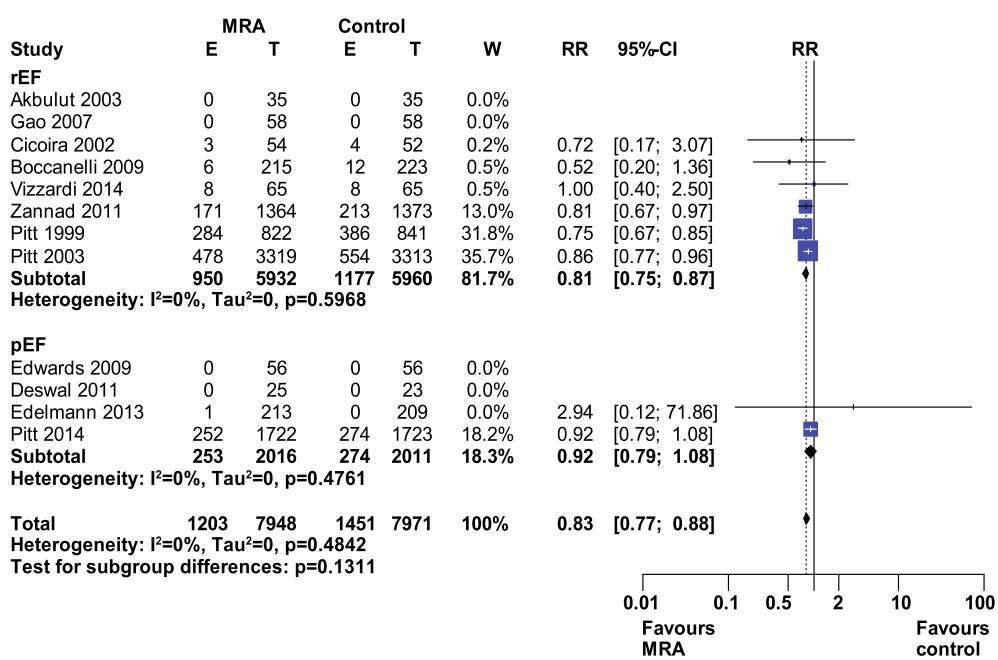

Fig. 3 Forest plot of all-cause mortality with MRA use in HF. Twelve trials reported all-cause mortality rates with MRA use in HF compared to control. Our Forest plot has been subdivided according to HF type

\section{Strengths and limitations}

Our systematic review has strengths including adherence to PRISMA reporting guidelines. In addition, our conclusions are based on evidence of moderate and high quality (GRADE). HFpEF evidence was of moderate quality, and HFrEF evidence was of high quality for cardiovascular death and all-cause mortality. The quality of evidence for cardiovascular death and all-cause mortality was reduced due the evidence for MRA use in HFpEF being limited to a single trial with large effect size [7], and several smaller trials with confidence intervals crossing unity [18, 27, 28]. For cardiovascular hospitalizations, the quality of evidence was reduced by confidence intervals in HFpEF and HFrEF studies crossing unity [7, 33]. Evidence for hyperkalemia and gynecomastia with MRA usage was of high quality. Overall, the evidence supporting MRA use in HFrEF is based on a larger number of trials with significant effect sizes for reducing adverse cardiac events. In contrast, the evidence for MRA use in HFpEF is based on a smaller number of trials, only one of which had a significant reduction in cardiovascular hospitalizations but no other adverse cardiac events [7]. Finally, our conclusions supporting MRA usage in HFrEF align with current American Heart Association guidelines which recommend MRAs for patients with HFrEF and NYHA class II-IV symptoms or following acute MI complicated by $\mathrm{HF}$ and $\mathrm{EF} \leq 40 \%$ [1].

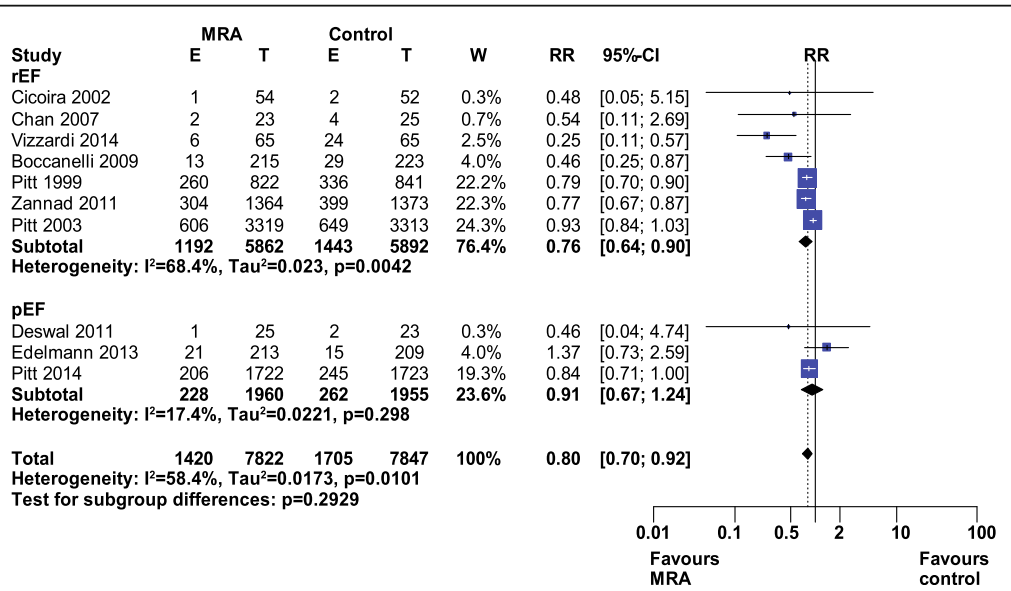

Fig. 4 Forest plot of cardiovascular hospitalizations with MRA use in HF. Ten trials reported cardiovascular hospitalization rates with MRA use in HF compared to control. Our Forest plot has been subdivided according to HF type 


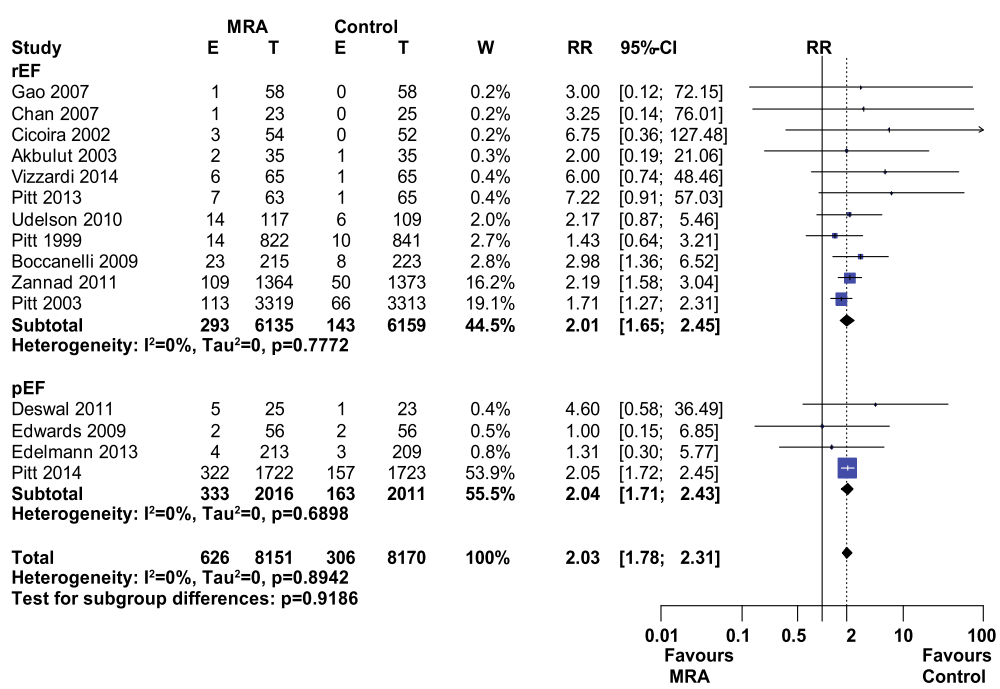

Fig. 5 Forest plot of hyperkalemia with MRA use in HF. Fifteen trials reported hyperkalemia rates with MRA use in HF compared to control. Our Forest plot has been subdivided according to HF type

\section{Implications}

Current guidelines suggest MRAs are useful in treating HFrEF and acute MI complicated by HF [1, 34]. We demonstrate that treatment of HFpEF with MRAs does not reduce adverse cardiac events. However, MRAs do cause harm from hyperkalemia (NNH 26 [20-34]) and gynecomastia (e.g., nonselective MRA, NNH 33 [19-63]). Selective MRAs offer a slight advantage in terms of no significant gynecomastia while having equivalent reductions in adverse cardiac outcomes. We suggest continued usage of MRAs in HFrEF, where there is a significant reduction in adverse cardiac outcomes, e.g., cardiovascular death (NNT 34 [26-50]), or allcause mortality (NNT 32 [24-45]). We suggest that MRAs be avoided in HFpEF as they do not reduce adverse cardiovascular outcomes.

\section{Conclusions}

Our systematic review provides evidence that MRAs should not be used in HFpEF. MRA usage in HFpEF provides a risk of hyperkalemia and/or gynecomastia without reducing adverse cardiac events. In contrast, MRA usage in HFrEF significantly reduces adverse cardiac events.

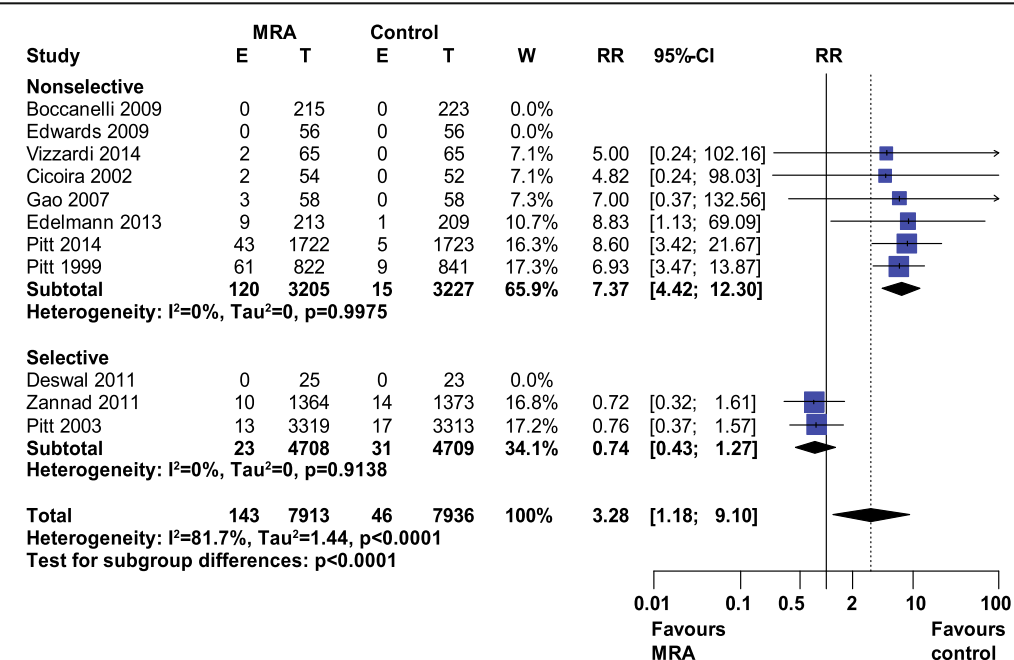

Fig. 6 Forest plot of gynecomastia with MRA use in HF. Eleven trials reported gynecomastia rates with MRA use in HF compared to control. Our Forest plot has been subdivided according to MRA type 


\section{Appendix 1}

Table 4 Ovid MEDLINE and EMBASE search strategy

\begin{tabular}{|c|c|c|}
\hline 1 & Exp heart failure/ & $376335 / 94836$ \\
\hline 2 & Exp Cardiomyopathy/ & $113241 / 78224$ \\
\hline 3 & Exp Ventricular dysfunction/ & $13819 / 28965$ \\
\hline 4 & $\begin{array}{l}\text { ((heart or cardiac or myocardial) adj2 } \\
\text { (failure or decompensation)).ti,ab }\end{array}$ & $211838 / 130125$ \\
\hline 5 & $\begin{array}{l}\text { ((congestive or chronic) adj2 heart } \\
\text { failure).ti,ab }\end{array}$ & $67554 / 45613$ \\
\hline 6 & $\begin{array}{l}\text { ((ventric\$) adj2 (failure or insufficien\$ or } \\
\text { dysfunction\$ or function\$)).ti,ab }\end{array}$ & $82523 / 54772$ \\
\hline 7 & $\begin{array}{l}\text { ((Reduced or preserved) adj2 "ejection } \\
\text { fraction").ti,ab. }\end{array}$ & $6293 / 2856$ \\
\hline 8 & (HFpEF or HFrEF).mp & $2216 / 776$ \\
\hline 9 & $\begin{array}{l}\text { ((diastol\$ or systol\$) adj2 ((failure or } \\
\text { dysfunction\$ or function\$)).ti,ab }\end{array}$ & $48968 / 27523$ \\
\hline 10 & Or/1-9 & $538115 / 275995$ \\
\hline 11 & (animal\$ not (human\$ and animal\$)).sh,hw. & $3987846 / 4137327$ \\
\hline 12 & 10 NOT 11 & $501141 / 241626$ \\
\hline 13 & exp Aldosterone Antagonist/ & $32899 / 8124$ \\
\hline 14 & $\begin{array}{l}\text { (eplerenone or inspra or spironolactone or } \\
\text { aldactone or aldo\$ or mineralocorticoid } \\
\text { receptor antagon } \$ \text { or canren\$ or fineren\$). } \\
\text { ti,ab }\end{array}$ & $134477 / 63667$ \\
\hline 15 & (aldosterone adj2 antagon\$). ti,ab & $7857 / 2066$ \\
\hline 16 & Or/13-15 & $134563 / 63667$ \\
\hline 17 & 16 NOT 11 & $111915 / 46794$ \\
\hline 18 & 12 and 17 & $20116 / 4880$ \\
\hline 19 & $\begin{array}{l}\left(1995^{*} \text { or } 1996^{*} \text { or } 1997^{*} \text { or } 1998^{*} \text { or } 1999^{*}\right. \\
\text { or } 2000^{*} \text { or } 2001^{*} \text { or } 2002^{*} \text { or } 2003^{*} \text { or } \\
2004^{*} \text { or } 2005^{*} \text { or } 2006^{*} \text { or } 2007^{*} \text { or } 2008^{*} \\
\text { or } 2009^{*} \text { or } 2010^{*} \text { or } 2011^{*} \text { or } 2012^{*} \text { or } \\
\left.2013^{*} \text { or } 2014^{*} \text { or } 2015^{*} \text { or } 2016^{*}\right) . d d\end{array}$ & $21741062 / 0$ \\
\hline 20 & $\begin{array}{l}\left(1995^{*} \text { or } 1996^{*} \text { or } 1997^{*} \text { or } 1998^{*} \text { or } 1999^{*}\right. \\
\text { or } 2000^{*} \text { or } 2001^{*} \text { or } 2002^{*} \text { or } 2003^{*} \text { or } \\
2004^{*} \text { or } 2005^{*} \text { or } 2006^{*} \text { or } 2007^{*} \text { or } 2008^{*} \\
\text { or } 2009^{*} \text { or } 2010^{*} \text { or } 2011^{*} \text { or } 2012^{*} \text { or } \\
\left.2013^{*} \text { or } 2014^{*} \text { or } 2015^{*} \text { or } 2016^{*}\right) \text {.ed }\end{array}$ & $0 / 16309819$ \\
\hline 21 & 19 or 20 & $21741062 / 16309819$ \\
\hline 22 & 18 AND 21 & $17787 / 3735$ \\
\hline 23 & $\begin{array}{l}\text { (book or conference paper or editorial or } \\
\text { letter or review).pt. not exp randomized } \\
\text { controlled trial/ }\end{array}$ & $4298634 / 3319843$ \\
\hline 24 & $\begin{array}{l}\text { (random sampl\$ or random digit\$ or } \\
\text { random effect\$ or random survey or } \\
\text { random regression).ti,ab. not exp } \\
\text { randomized controlled trial/ }\end{array}$ & $69917 / 56001$ \\
\hline 25 & $\begin{array}{l}\text { (random\$ or placebo\$ or single blind\$ or } \\
\text { double blind\$ or triple blind\$).ti,ab. }\end{array}$ & $1177770 / 884750$ \\
\hline 26 & 25 not ( 23 or 24 or 11$)$ & $908857 / 648772$ \\
\hline 27 & 22 and 26 & $1802 / 503=2305$ \\
\hline 28 & Remove duplicates from 27 & $1372 / 502=1874$ \\
\hline
\end{tabular}

(Search performed January 29, 2016)

\section{Appendix 2}

Table 5 Cochrane database search strategy

\begin{tabular}{|c|c|c|}
\hline 1 & exp heart failure & 1412 \\
\hline 2 & exp Cardiomyopathy & 133 \\
\hline 3 & exp Ventricular dysfunction & 225 \\
\hline 4 & $\begin{array}{l}\text { ((heart or cardiac or myocardial) near/2 } \\
\text { (failure or decompensation)):ti,ab,kw }\end{array}$ & 14941 \\
\hline 5 & $\begin{array}{l}\text { ((congest* or chronic) near/2 "heart } \\
\text { failure"):ti,ab,kw }\end{array}$ & 6158 \\
\hline 6 & $\begin{array}{l}\text { (ventric* near (fail* or insufficien* or } \\
\text { dysfunction* or function*)):ti,ab,kw }\end{array}$ & 9067 \\
\hline 7 & $\begin{array}{l}\text { ((diastol* or systol*) near/2 (failure or } \\
\text { dysfunction* or function*)):ti, ab, kw }\end{array}$ & 2866 \\
\hline 8 & $\begin{array}{l}\text { ((Reduced or preserved) near/2 } \\
\text { (ejection or fraction or "EF")):ti, ab, kw }\end{array}$ & 532 \\
\hline 9 & ("HFpEF" or "HFrEF"):ti, ab, kw & 161 \\
\hline 10 & $\begin{array}{l}\# 1 \text { or } \# 2 \text { or } \# 3 \text { or } \# 4 \text { or } \# 5 \text { or } \# 6 \text { or \#7 } \\
\text { or \#8 or \#9 }\end{array}$ & 21983 \\
\hline 11 & exp Aldosterone Antagonist* & 44 \\
\hline 12 & (mineralocorticoid receptor antagonist*):ti,ab, kw & 486 \\
\hline 13 & $\begin{array}{l}\text { (eplerenone or inspra or spironolactone or } \\
\text { aldactone or canren* or fineren*):ti,ab,kw }\end{array}$ & 1309 \\
\hline 14 & Aldosterone near/2 antagon* & 393 \\
\hline 15 & $\# 11$ or \#12 or \#13 & 1661 \\
\hline 16 & $\# 10$ and \#15 & 692 \\
\hline
\end{tabular}

Updated Jan-29-2016

\section{Additional files}

Additional file 1: Figure S1. Forest plot of cardiovascular death in HF with MRA use by MRA type. (EPS $2561 \mathrm{~kb}$ )

Additional file 2: Figure S2. Forest plot of all-cause mortality in HF with MRA use by MRA type. (EPS $2763 \mathrm{~kb}$ )

Additional file 3: Figure S3. Forest plot of cardiovascular hospitalization with MRA use by MRA type. (EPS $2740 \mathrm{~kb}$ )

Additional file 4: Figure S4. Forest plot of hyperkalemia with MRA use by MRA type. (EPS $2919 \mathrm{~kb}$ )

Additional file 5: Figure S5. Funnel plot of cardiovascular death with MRA use. (EPS $737 \mathrm{~kb}$ )

Additional file 6: Figure S6. Funnel plot of all-cause mortality with MRA use. (EPS $736 \mathrm{~kb}$ )

Additional file 7: Figure S7. Funnel plot of cardiovascular hospitalizations with MRA use. (EPS $710 \mathrm{~kb}$ )

Additional file 8: Figure S8. Funnel plot of hyperkalemia with MRA use. (EPS $736 \mathrm{~kb}$ )

Additional file 9: Figure S9. Funnel plot of gynecomastia with MRA use. (EPS $743 \mathrm{~kb}$ )

\section{Abbreviations}

HF: Heart failure; HFpEF: Heart failure with preserved ejection fraction; HFrEF: Heart failure with reduced ejection fraction; MI: Myocardial infarction; MRA: Mineralocorticoid receptor antagonist; NNH: Number needed to harm; NNT: Number needed to treat; NYHA: New York Heart Association;

$\mathrm{RCT}$ : Randomized controlled trial; RR: Relative risk 


\section{Acknowledgements}

None.

\section{Funding}

There was no funding for this study.

\section{Availability of data and materials}

The datasets generated and analyzed in this study are available from the corresponding author on request.

\section{Authors' contributions}

NB: study conception and design, data acquisition, analysis, and interpretation, drafted manuscript. MM: study conception and design, data acquisition, analysis, interpretation, drafted manuscript. Both authors read and approved the final manuscript

\section{Competing interests}

The authors declare that they have no competing interests.

\section{Consent for publication}

Not applicable.

\section{Ethics approval and consent to participate}

Not applicable.

\section{Received: 5 August 2016 Accepted: 25 November 2016}

\section{Published online: 01 December 2016}

\section{References}

1. Yancy CW, Jessup M, Bozkurt B, Butler J, Casey Jr DE, Drazner MH, Fonarow GC, Geraci SA, Horwich T, Januzzi JL, Johnson MR, Kasper EK, Levy WC, Masoudi FA, McBride PE, McMurray JJ, Mitchell JE, Peterson PN Riegel B, Sam F, Stevenson LW, Tang WH, Tsai EJ, Wilkoff BL. 2013 ACCF/AHA guideline for the management of heart failure: a report of the American College of Cardiology Foundation/American Heart Association Task Force on practice guidelines. Circulation. 2013;128(16):e240-327.

2. Cheng JW, Nayar M. A review of heart failure management in the elderly population. Am J Geriatr Pharmacother. 2009;7(5):233-49.

3. Bhuiyan T, Maurer MS. Heart failure with preserved ejection fraction: persistent diagnosis, therapeutic enigma. Curr Cardiovasc Risk Rep. 2011;5(5):440-9.

4. Flather MD, Shibata MC, Coats AJ, Van Veldhuisen DJ, Parkhomenko A, Borbola J, Cohen-Solal A, Dumitrascu D, Ferrari R, Lechat P, Soler-Soler J, Tavazzi L, Spinarova L, Toman J, Bohm M, Anker SD, Thompson SG, Poole-Wilson PA. Randomized trial to determine the effect of nebivolo on mortality and cardiovascular hospital admission in elderly patients with heart failure (SENIORS). Eur Heart J. 2005;26(3):215-25

5. Yusuf S, Pfeffer MA, Swedberg K, Granger CB, Held P, McMurray Michelson EL, Olofsson B, Ostergren J. Effects of candesartan in patients with chronic heart failure and preserved left-ventricular ejection fraction: the CHARM-Preserved Trial. Lancet. 2003;362(9386):777-81.

6. Cleland JG, Tendera M, Adamus J, Freemantle N, Polonski L, Taylor J. The perindopril in elderly people with chronic heart failure (PEP-CHF) study. Eur Heart J. 2006;27(19):2338-45.

7. Pitt B, Pfeffer MA, Assmann SF, Boineau R, Anand IS, Claggett B, Clausell N, Desai AS, Diaz R, Fleg JL, Gordeev I, Harty B, Heitner JF, Kenwood CT, Lewis EF, O'Meara E, Probstfield JL, Shaburishvili T, Shah SJ, Solomon SD, Sweitzer NK Yang S, McKinlay SM, TOPCAT I. Spironolactone for heart failure with preserved ejection fraction. N Engl J Med. 2014:370(15):1383-92.

8. Komajda M, Lam CS. Heart failure with preserved ejection fraction: a clinical dilemma. Eur Heart J. 2014:35(16):1022-32.

9. Meta-analysis Global Group in Chronic Heart Failure (MAGGIC). The survival of patients with heart failure with preserved or reduced left ventricular ejection fraction: an individual patient data meta-analysis. European Heart Journal 2012 July;33(14):1750-7.

10. de Gasparo M, Joss U, Ramjoue HP, Whitebread SE, Haenni H, Schenkel L, Kraehenbuehl C, Biollaz M, Grob J, Schmidlin J. Three new epoxyspirolactone derivatives: characterization in vivo and in vitro. J Pharmacol Exp Ther. 1987;240(2):650-6.

11. Pitt B, Zannad F, Remme WJ, Cody R, Castaigne A, Perez A, Palensky J Wittes $J$. The effect of spironolactone on morbidity and mortality in patients with severe heart failure. Randomized Aldactone Evaluation Study Investigators. N Engl J Med. 1999:341(10):709-17.

12. Pitt B, Remme W, Zannad F, Neaton J, Martinez F, Roniker B, Bittman R, Hurley S, Kleiman J, Gatlin M. Eplerenone Post-Acute Myocardial Infarction Heart Failure Efficacy and Survival Study Investigators. Eplerenone, a selective aldosterone blocker, in patients with left ventricular dysfunction after myocardial infarction. N Engl J Med. 2003;348(14):1309-21. Erratum appears in N Engl J Med. 2003 May 29;348(22):2271.

13. Ikram H, Webster MW, Nicholls MG, Lewis GR, Richards AM, Crozier IG Combined spironolactone and converting-enzyme inhibitor therapy for refractory heart failure. Aust N Z J Med. 1986;16(1):61-3.

14. Pitt B, Zannad F, Remme WJ, Cody R, Castaigne A, Perez A, Palensky J, Wittes $J$. The effect of spironolactone on morbidity and mortality in patients with severe heart failure. N Engl J Med. 1999;341(10):02.

15. Brilla CG, Pick R, Tan LB, Janicki JS, Weber KT. Remodeling of the rat right and left ventricles in experimental hypertension. Circ Res. 1990;67(6):1355-64

16. Chen $Y$, Wang H, Lu Y, Huang X, Liao Y, Bin J. Effects of mineralocorticoid receptor antagonists in patients with preserved ejection fraction: a meta-analysis of randomized clinical trials. BMC Med. 2015:13:10.

17. Lloyd SJ, Mauro VF. Spironolactone in the treatment of congestive heart failure. Ann Pharmacother. 2000;34(11):1336-40 [Review] [20 refs].

18. Edelmann F, Wachter R, Schmidt AG, Kraigher-Krainer E, Colantonio C, Kamke W, Duvinage A, Stahrenberg R, Durstewitz K, Loffler M, Dungen HD, Tschope C, Herrmann-Lingen C, Halle M, Hasenfuss G, Gelbrich G, Pieske B, Aldo-DHF I. Effect of spironolactone on diastolic function and exercise capacity in patients with heart failure with preserved ejection fraction: the Aldo-DHF randomized controlled trial. JAMA. 2013;309(8):781-91.

19. Moher D, Liberati A, Tetzlaff J, Altman DG. Preferred reporting items for systematic reviews and meta-analyses: the PRISMA statement. Int J Surg. 2010:8(5):336-41.

20. Guyatt GH, Oxman AD, Vist GE, Kunz R, Falck-Ytter Y, Alonso-Coello P, Schunemann HJ. GRADE: an emerging consensus on rating quality of evidence and strength of recommendations. BMJ. 2008;336(7650):924-6.

21. Schwarzer G. meta: A n R package for meta-analysis. R News. 2007;7(3):40 45

22. R Core Team. R: A Language and Environment for Statistical Computing [computer program]. Vienna: R Foundation for Statistical Computing; 2015. http://www.Rproject.org/.

23. Akbulut M, Ozbay Y, Ilkay E, Karaca I, Arslan N. Effects of spironolactone and metoprolol on QT dispersion in heart failure. Jpn Heart J. 2003:44(5):681-92.

24. Boccanelli A, Mureddu GF, Cacciatore G, Clemenza F, Di LA, Gavazzi A, Porcu M, Latini R, Lucci D, Maggioni AP, Masson S, Vanasia M, de Simone G, AREA INC. Anti-remodelling effect of canrenone in patients with mild chronic heart failure (AREA IN-CHF study): final results. Eur J Heart Fail. 2009:11(1):68-76

25. Chan AK, Sanderson JE, Wang T, Lam W, Yip G, Wang M, Lam YY, Zhang Y, Yeung L, Wu EB, Chan WW, Wong JT, So N, Yu CM. Aldosterone receptor antagonism induces reverse remodeling when added to angiotensin receptor blockade in chronic heart failure. J Am Coll Cardiol. 2007:50(7):591-6.

26. Cicoira M, Zanolla L, Rossi A, Golia G, Franceschini L, Brighetti G, Marino P, Zardini P. Long-term, dose-dependent effects of spironolactone on left ventricular function and exercise tolerance in patients with chronic heart failure J Am Coll Cardiol. 2002:40(2):304-10

27. Deswal A, Richardson P, Bozkurt B, Mann DL. Results of the Randomized Aldosterone Antagonism in Heart Failure with Preserved Ejection Fraction trial (RAAM-PEF). J Card Fail. 2011;17(8):634-42.

28. Edwards NC, Steeds RP, Stewart PM, Ferro CJ, Townend JN. Effect of spironolactone on left ventricular mass and aortic stiffness in early-stage chronic kidney disease: a randomized controlled trial. J Am Coll Cardiol. 2009:54:505-12

29. Gao X, Peng L, Adhikari CM, Lin J, Zuo Z. Spironolactone reduced arrhythmia and maintained magnesium homeostasis in patients with congestive heart failure. J Card Fail. 2007;13(3):170-7

30. Pitt B, Kober L, Ponikowski P, Gheorghiade M, Filippatos G, Krum H, Nowack C, Kolkhof P, Kim SY, Zannad F. Safety and tolerability of the novel non-steroidal mineralocorticoid receptor antagonist BAY 94-8862 in patients with chronic heart failure and mild or moderate chronic kidney disease: a randomized, double-blind trial. Eur Heart J. 2013;34(31):2453-63.

31. Udelson JE, Feldman AM, Greenberg B, Pitt B, Mukherjee R, Solomon HA, Konstam MA. Randomized, double-blind, multicenter, placebo-controlled 
study evaluating the effect of aldosterone antagonism with eplerenone on ventricular remodeling in patients with mild-to-moderate heart failure and left ventricular systolic dysfunction. Circ Heart Fail. 2010;3(3):347-53.

32. Vizzardi E, Nodari S, Caretta G, D'Aloia A, Pezzali N, Faden G, Lombardi C, Raddino R, Metra M, Dei CL. Effects of spironolactone on long-term mortality and morbidity in patients with heart failure and mild or no symptoms. Am J Med Sci. 2014;347(4):271-6.

33. Zannad F, McMurray JJ, Krum H, Van Veldhuisen DJ, Swedberg K, Shi H, Vincent J, Pocock SJ, Pitt B, EMPHASIS-HF Study Group. Eplerenone in patients with systolic heart failure and mild symptoms. N Engl J Med. 2011:364(1):11-21

34. James PA, Oparil S, Carter BL, Cushman WC, Dennison-Himmelfarb C, Handler J, Lackland DT, LeFevre ML, Mackenzie TD, Ogedegbe O, Smith Jr SC, Svetkey LP, Taler SJ, Townsend RR, Wright Jr JT, Narva AS, Ortiz E. 2014 evidence-based guideline for the management of high blood pressure in adults: report from the panel members appointed to the Eighth Joint National Committee (JNC 8). JAMA. 2014:311(5):507-20.

Submit your next manuscript to BioMed Central and we will help you at every step:

- We accept pre-submission inquiries

- Our selector tool helps you to find the most relevant journal

- We provide round the clock customer support

- Convenient online submission

- Thorough peer review

- Inclusion in PubMed and all major indexing services

- Maximum visibility for your research

Submit your manuscript at www.biomedcentral.com/submit
Biomed Central 